\title{
HLA antigens in psoriasis and psoriatic arthritis
}

\author{
J C WOODROW AND A ILCHYSYN* \\ From the Departments of Medicine and Dermatology, Royal Liverpool Hospital, Liverpool L69 3BX.
}

SUMmARY HLA phenotypes were determined in 50 patients with psoriasis alone and in 50 patients with psoriasis and psoriatic arthritis. Positive associations were found in both groups with B13, B17, B37, Cw6, and DR7, and in addition with C4A6. Higher relative risks were found in respect to the patients with psoriasis alone compared with those with arthritis, and this suggests the involvement of additional genetic factors predisposing to peripheral arthritis. In patients with psoriasis only, the presence of $\mathrm{Cw} 6$ was associated with a significantly earlier age of onset.

Analysis of the pattern of familial aggregation of psoriasis suggested the likelihood that several genetic loci might be involved in producing increased susceptibility. ${ }^{12}$ That HLA linked loci are involved was shown by the demonstration of positive associations between psoriasis and several HLA antigens, and in particular with B17, B13, Cw6, and DR7.3-9 Evidence for familial aggregation of psoriatic arthritis $^{10}$ and for the association of the presence of arthritis with HLA-B27 $7^{11}$ and possibly other HLA antigens ${ }^{12}$ suggested that additional genetic factors might predispose to the development of joint disease.

The present study was aimed at examining further the relationship between the HLA-A, B, C, and DR polymorphisms in groups of patients with psoriasis only or with psoriasis and peripheral psoriatic arthritis, together with a study of the $\mathrm{C} 4$ polymorphism (the C4A and C4B loci being closed linked to the HLA-B and DR loci). Evidence has previously been put forward for heterogeneity in relation to age of onset of psoriasis, ${ }^{411}$ and this was looked at again in the light of the more recently discovered associations.

\section{Patients and methods}

Fifty successive patients with psoriasis vulgaris but without evidence of peripheral psoriatic arthritis were ascertained in a dermatological outpatient department. A further 50 patients with psoriasis and with a chronic peripheral arthritis judged to be of a clinical pattern known to be associated with psoriasis (groups 1,2, 3, and 4 in a previous study of psoriatic arthritis ${ }^{11}$ ) were ascertained in a rheumatological unit.

Control subjects were laboratory and nursing staff

*Present address: The General Hospital, Stoke-on-Trent.

Received for publication 3 July 1985.

Accepted for publication 2 August 1985 and medical students residing in the same geographical area as the patients. HLA-A, B, and C typing was carried out by a two stage lymphocytotoxicity microassay ${ }^{13}$ and HLA-DR typing was performed using an adaptation of the two colour fluorescence method. ${ }^{14} \mathrm{C} 4$ phenotyping was carried out by electrophoresis and immunofixation. ${ }^{15}$ Statistical analysis was carried out using Woolf's method ${ }^{16}$ with modification by Haldane ${ }^{17}$ and, in the case of the comparison of age of onset distributions, by theo Wilcoxon rank sum test.

\section{Results}

The data obtained from testing patients and controls for HLA-A and B locus antigens are shown in table 1a and for HLA-C and DR antigens in table $1 \mathrm{~b}$.

With regard to $\mathrm{C} 4 \mathrm{~A}$ and $\mathrm{C} 4 \mathrm{~B}$ phenotyping, the notable finding was that 12 out of 105 controls $(11.4 \%)$ typed as C4A6 compared with 19 out of 50 psoriatic $(38 \%)$ and 11 out of $50(22 \%)$ psoriatic arthritic patients. The excess of $\mathrm{C} 4 \mathrm{~A} 6$ in psoriatic patients was associated with a probability of $\mathrm{p}=0.0003$.

Table 2 gives the relative risks for both groups of patients for various phenotypes which showed positive associations.

The results support the previously shown positive associations of psoriasis with HLA-B13, B17, B37, Cw6, and DR7. The previously reported association of psoriatic arthritis with B27 was confirmed. C4A6 was found to be significantly associated with psoriasis with a relative risk of 4.63 , the risk being somewhat lower in the case of psoriatic arthritis. The various positive associations with antigens determined at the different loci point to certain haplotypes being associated with increased liability to disease, C4A6 being involved in these haplotypes. The association of $\mathrm{C} 4 \mathrm{~A} 6$ in haplotypes 
TABLE 1a Frequencies of HLA-A and B phenotypes in the two groups of patients and in controls.

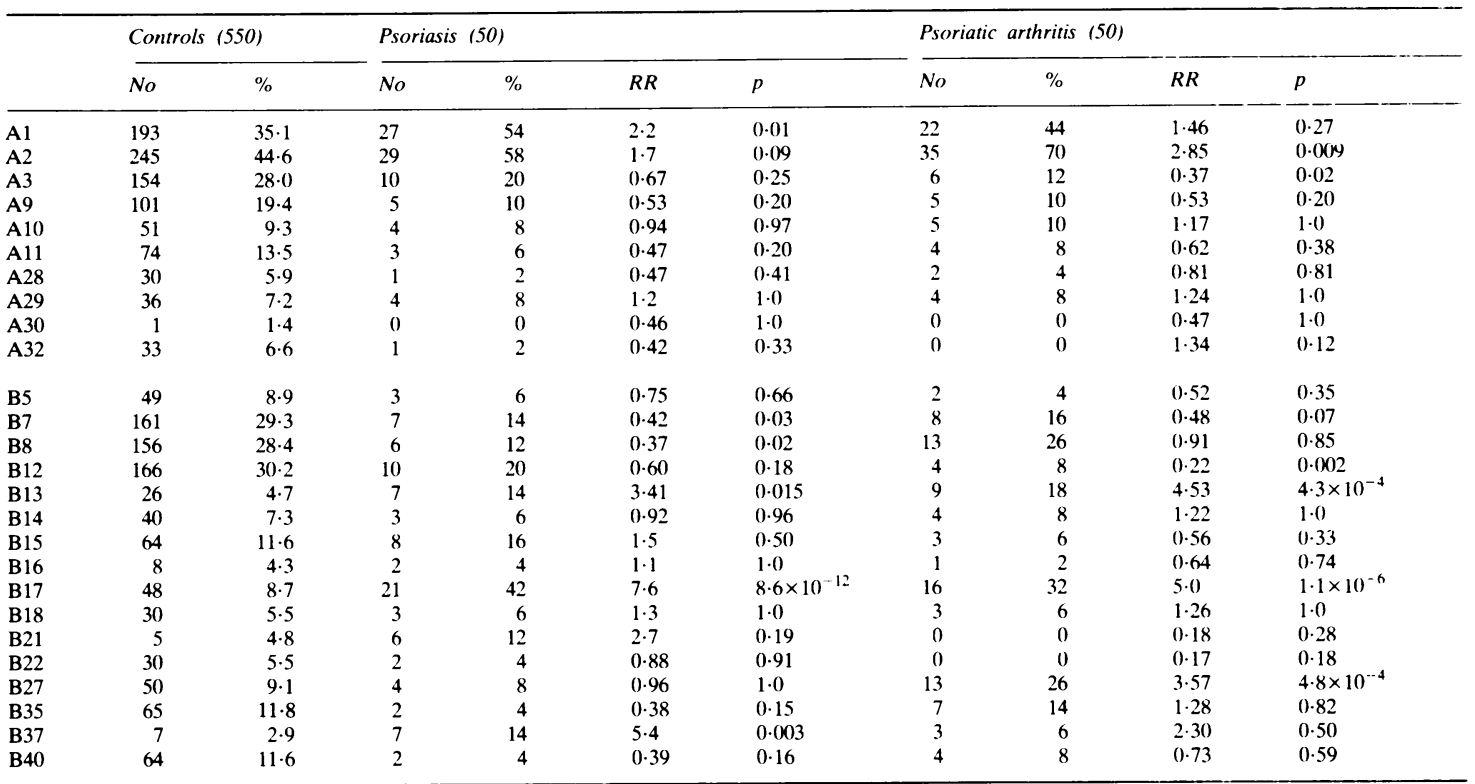

TABLE 1b Frequencies of HLA-C and DR phenotypes in the two groups of patients and in controls.

\begin{tabular}{|c|c|c|c|c|c|c|c|c|c|c|}
\hline & \multicolumn{2}{|c|}{ Controls (115) } & \multicolumn{4}{|c|}{ Psoriasis (50) } & \multicolumn{4}{|c|}{ Psoriatic arthritis (50) } \\
\hline & No & $\%$ & No & $\%$ & $R R$ & $p$ & No & $\%$ & $R R$ & $p$ \\
\hline Cw1 & 13 & $11 \cdot 3$ & 3 & 6 & $0 \cdot 56$ & 0.44 & 9 & 18 & 1.74 & $0 \cdot 36$ \\
\hline Cw2 & 3 & $2 \cdot 6$ & 3 & 6 & $2 \cdot 37$ & 0.54 & 6 & 12 & $4 \cdot 70$ & $0 \cdot 04$ \\
\hline Cw3 & 30 & $26 \cdot 1$ & 12 & 24 & 0.91 & 0.93 & 7 & 14 & 0.48 & $0 \cdot 13$ \\
\hline Cw4 & 19 & $16 \cdot 5$ & 2 & 4 & $0 \cdot 26$ & $0 \cdot 05$ & 6 & 12 & 0.72 & $0 \cdot 61$ \\
\hline Cw5 & 19 & $16 \cdot 5$ & 6 & 12 & 0.72 & 0.61 & 7 & 14 & $0 \cdot 85$ & 0.86 \\
\hline Cw6 & 27 & $23 \cdot 5$ & 36 & 72 & $8 \cdot 10$ & $1 \times 10^{-8}$ & 25 & 50 & $3 \cdot 22$ & 0.0014 \\
\hline \multirow[t]{2}{*}{ Cw7 } & 48 & $41 \cdot 7$ & 17 & 34 & 0.73 & $0 \cdot 45$ & 15 & 30 & $0 \cdot 61$ & $0 \cdot 21$ \\
\hline & \multicolumn{10}{|c|}{ Controls (168) } \\
\hline DR1 & 32 & $19 \cdot 1$ & 8 & 16 & 0.84 & 0.78 & 11 & 22 & $1 \cdot 22$ & 0.80 \\
\hline DR2 & 48 & $28 \cdot 6$ & 14 & 28 & 0.99 & $1 \cdot 0$ & 7 & 14 & 0.43 & 0.06 \\
\hline DR3 & 44 & $26 \cdot 2$ & 6 & 12 & 0.41 & 0.06 & 16 & 32 & $1 \cdot 34$ & 0.53 \\
\hline DR4 & 59 & $35 \cdot 1$ & 16 & 32 & $0 \cdot 88$ & $0 \cdot 81$ & 16 & 32 & $0 \cdot 88$ & $0 \cdot 81$ \\
\hline DR5 & 21 & $12 \cdot 5$ & 5 & 10 & 0.83 & 0.82 & 5 & 10 & 0.83 & 0.82 \\
\hline DR6 & 26 & $15 \cdot 5$ & 8 & 16 & $1 \cdot 08$ & $1 \cdot 0$ & 18 & 36 & $3 \cdot 06$ & 0.003 \\
\hline DR7 & 49 & $29 \cdot 2$ & 31 & 62 & 3.90 & $4.9 \times 10^{-5}$ & 17 & 34 & $1 \cdot 26$ & 0.63 \\
\hline
\end{tabular}

TABLE 2 Relative risk ${ }^{16} 17$ for various $H L A$ phenotypes in patients with psoriasis alone and with psoriatic arthritis.

\begin{tabular}{lll}
\hline & Psoriasis & Psoriatic arthritis \\
\hline B13 & $3 \cdot 4$ & $4 \cdot 5$ \\
B17 & $7 \cdot 6$ & $5 \cdot 0$ \\
B37 & $5 \cdot 4$ & $2 \cdot 3$ \\
Cw6 & $8 \cdot 1$ & $3 \cdot 2$ \\
DR7 & $3 \cdot 9$ & $1 \cdot 3$ \\
C4A6 & $4 \cdot 6$ & $3 \cdot 2$ \\
B27 & $0 \cdot 96$ & $3 \cdot 6$ \\
\hline
\end{tabular}

involving B17 and DR7 was suggested in the case of the normal population by Awdeh et $a l^{18}$ and is strongly supported by the present study. Ten out of 12 C4A6 positive controls were also Cw6 positive, compared to nine out of 93 C4A6 negative subjects. Eight of the 10 were B17 positive and seven also DR7 positive. It appears likely, therefore, that B17, Cw6, DR7, and C4A6 are in linkage disequilibrium. Of the 19 C4A6 patients with psoriasis only, all were Cw6 positive and, of these, 18 were also B17 positive and one B13 positive, and 16 were DR7 
positive. In the case of patients with psoriatic arthritis, 11 were C4A6 positive, of whom nine were Cw6 positive, all were $\mathrm{B} 17$ positive, and eight were DR7 positive.

It is to be noted from table 2 that for each phenotype showing a positive association with psoriasis, with the exception of B13, the relative risk was lower in the case of psoriatic arthritis. Taking the risks for $\mathrm{Cw} 6$ as an example, the difference between $8 \cdot 2$ and $3 \cdot 2$ was significant $(p=0 \cdot 04)$.

\section{AGE OF ONSET}

Table 3 shows the statistics relating to the age at onset of psoriasis for the various subgroups. It is seen that when all the patients are considered together, Cw6 positive subjects tend to have an earlier age of onset than those negative for this antigen $(p=0 \cdot 015)$. Analysis of the two patient groups separately shows that the difference is only apparent in the group with psoriasis alone $(p=0 \cdot 013)$. There is a tendency for the age of onset of psoriasis to be somewhat earlier in the psoriasis only group, irrespective of Cw6, but the difference is not significant.

\section{Discussion}

It appears likely that haplotypes involving three $\mathrm{B}$ locus antigens (B13, B17, and B37), Cw6, DR7, and C4A6 are the ones that particularly carry genes involved in susceptibility to psoriasis. Linkage disequilibria between these phenotypes have been established from normal population data. ${ }^{18} 19$

An unsolved problem is to determine from the association data which of the loci showing positive associations is either directly involved in providing disease susceptibility genes or is the most closely linked to the susceptibility gene locus. Thus, it is possible that Cw6 itself is the product of the disease susceptibility gene or it may be in strong linkage

TABLE 3 Comparison of age of onset of psoriasis between various subgroups.

\begin{tabular}{|c|c|c|c|c|}
\hline & & \multicolumn{3}{|c|}{ Age of onset $(y)$} \\
\hline & & Mean & Median & $p^{*}$ \\
\hline All patients & $\begin{array}{l}\text { Cw6 positive } \\
\text { Cw6 negative }\end{array}$ & $\begin{array}{l}32 \cdot 2 \\
23 \cdot 7\end{array}$ & $\begin{array}{l}25 \cdot 0 \\
18 \cdot 0\end{array}$ & $0 \cdot 0153$ \\
\hline Psoriasis alone & $\begin{array}{l}\text { Cw6 positive } \\
\text { Cw6 negative }\end{array}$ & $\begin{array}{l}34 \cdot 4 \\
21 \cdot 3\end{array}$ & $\begin{array}{l}25 \cdot 5 \\
16 \cdot 0\end{array}$ & $0 \cdot 013$ \\
\hline \multirow[t]{2}{*}{ Psoriatic arthritis } & $\begin{array}{l}\text { Cw6 positive } \\
\text { Cw6 negative }\end{array}$ & $\begin{array}{l}30 \cdot 7 \\
27 \cdot 1\end{array}$ & $\begin{array}{l}25 \cdot 0 \\
25 \cdot 0\end{array}$ & 0.49 \\
\hline & $\begin{array}{l}\text { Psoriasis alone } \\
\text { Psoriatic arthritis }\end{array}$ & $\begin{array}{l}25 \cdot 1 \\
28 \cdot 9\end{array}$ & $\begin{array}{l}17 \cdot 5 \\
25 \cdot 0\end{array}$ & $0 \cdot 16$ \\
\hline
\end{tabular}

*Wilcoxon rank sum test. disequilibrium with that gene. Theoretical considerations suggest that the phenotype showing the strongest association is at the locus closest to the disease susceptibility gene locus. The problem arises as to how best to measure the strength of association and what genetic interpretation to give to it.

Bengtsson and Thomson ${ }^{20}$ have discussed this problem and suggested that a measure of 'attributable risk', signified as $\delta$, was superior in the present context than the relative risk as measured by the cross product ratio. They suggested that $\delta$ can be used as an estimate of the normalised linkage disequilibrium between the HLA phenotype and the disease susceptibility gene and that comparison of the values obtained for various HLA phenotypes could allow one to decide which has the strongest association from the genetic point of view.

Table 4 gives the $\delta$ values calculated from the data in the present study. In general they follow a similar pattern to that seen when the odds ratios are tabulated (table 2), that is, the highest values for both $\delta$ and the odds ratio are those for Cw6. A combined analysis of the data from five recently published studies in addition to the present one shows a higher relative risk for $\mathrm{B} 13, \mathrm{~B} 17, \mathrm{Cw} 6$, and DR7 in patients with psoriasis alone than in those with arthritis in addition. ${ }^{21}$

The reason for these differences probably lies in the likelihood that the genetic factors predisposing to the psoriasis is the same for both groups but that? important additional genetic factors are operating in the cases of those with arthritis. Therefore, the role of psoriasis inducing genes relative to the total genetic predisposition is relatively smaller in the arthritis patients, leading to lower relative risk and lower values for $\delta$. The fact that HLA-B27 is associated with an approximately fourfold increase in relative risk for psoriatic arthritis will of itself lead to some reduction in the relative risk for other $\mathrm{B}$ locus antigens, but it is very unlikely that this accounts for the finding. Evidence has been put forward for the splits of HLA-Bw16 (B38 and B39) being involved in increased susceptibility for psoria-

TABLE 4 Values for $\delta^{*}$ for various HLA antigens in the two disease groups.

\begin{tabular}{lcl}
\hline & Psoriasis & Psoriatic arthritis \\
\hline B13 & $0 \cdot 1$ & 0.035 \\
B17 & 0.365 & $0 \cdot 255$ \\
B37 & $0 \cdot 156$ & 0.021 \\
Cw6 & 0.634 & 0.346 \\
DR7 & 0.463 & $0 \cdot 068$ \\
C4A6 & $0 \cdot 3$ & $0 \cdot 119$ \\
B27 & -0.01 & $0 \cdot 186$ \\
\hline \multirow{2}{*}{$\delta=\frac{d-p}{1-p}$ where $d$ is the frequency of the antigen in the disease group and $p$}
\end{tabular}


tic arthritis, ${ }^{12}{ }^{22-25}$ but the present study and presumably the study of Armstrong et $a l^{26}$ provide no evidence to support this suggestion and there is considerable heterogeneity between the different series. There remain additional genetic factors to be discovered which predispose to psoriatic arthritis and these may be HLA linked or unlinked.

\section{AGE OF ONSET}

Previous studies showed good evidence that patients with psoriasis who were B13 and particularly B17 positive were more likely to have a clinical onset in the first two decades than those negative for these antigens. ${ }^{45}$ There is support for this in the present study in which, when one includes all the patients, the median age of onset of psoriasis is lower for the Cw6 positive subjects than for those negative for this antigen. This difference, however, only applied to the group with psoriasis alone. The age of onset distribution in the case of patients with psoriatic arthritis was no different in Cw6 positive from that in Cw6 negative patients. It is possible to speculate that other genetic factors, possibly non-HLA linked, predispose to both psoriasis and psoriatic arthritis, and the role of Cw6 (or of a gene in linkage disequilibrium with Cw6) in determining a relatively early age of onset of psoriasis is relatively speaking reduced.

It is very likely that psoriasis is genetically heterogeneous and that various environmental triggers interact with the different genotypes involved. Thus an earlier onset may be the result of factors, such as infection, having the liability to precipitate the onset of psoriasis in the earlier decades in the presence of particular HLA linked genes.

We are grateful to our dermatological colleagues for allowing us to study their patients with psoriasis.

\section{References}

${ }^{1}$ Lomholt G. Environment and genetics in psoriasis. In: Farber EM, Cox AS, eds. Psoriasis. Proceedings of the International Symposium, Stanford University. Stanford, California: Stanford University Press, 1971:21-4.

2 Watson W, Cann HM, Farber EM, Nall ML. Genetics of psoriasis-family study. In: Farber EM, Cox AS, eds. Psoriasis. Proceedings of the International Symposium. Stanford University. Stanford. California: Stanford University Press, 1971:16-19.

${ }^{3}$ White SH, Newcombe VD, Mickey MR. Terasaki PI. Disturbance of HL-A angtigen frequency in psoriasis. $N$ Engl $J$ Med 1972;287:740-3.

4 Svejgaard A. Stahl-Nielsen LS, Svejgaard E, et al. HLA in psoriasis vulgaris and in pustular psoriasis: population and family studies. Br J Dermatol 1974;91:145-153.
5 Woodrow JC, Dave VK. Usher N, Anderson J. The HL-A system and psoriasis. Br J Dermatol 1975;92:427-36.

${ }^{6}$ Tsuji K. Inouye H. Nose Y, et al. Further study of HLA-A, B. C. D. DR and haplotype antigen frequencies in psoriasis vulgaris. Acta Derm Venereol /Suppl/ (Stockh) 1979;87:107-8.

7 Tiilikainen A. Lassus A. Karvonen J, et al. Psoriasis and HLA-Cw6. Br J Dermatol 1980:102:179-84.

${ }^{\rtimes}$ Marcusson JA, Johannesson A. Moller E. HLA-A, B, C, and DR antigens in psoriasis. Tissue Antigens 1981;17:525-9.

${ }^{9}$ Hawkins BR. Tiwari JL, Lowe N, et al. HLA and psoriasis. In: Terasaki PI, ed. Histocompatibility testing. Los Angeles: UCLA Tissue Typing Laboratory. 1980:711-4.

${ }^{10}$ Moll JMH. Wright V. Familial occurrence of psoriatic arthritis. Ann Rheum Dis 1973:32:181-201.

1 Eastmond CJ. Woodrow JC. The HLA system and the arthropathies associated with psoriasis. Ann Rheum Dis 1977;36: 112-20.

12 Espinoza LR, Vasey FB, Oh JH. et al. Association between HLA-Bw38 and peripheral psoriatic arthritis. Arthritis Rheum 1978:21:72-5.

13 Terasaki PI. McClelland JD. Microdroplet assay of human serum cytotoxins. Nature 1964:204:998-1000.

14 van Rood JJ, van Leeuwen A. Ploem JS. Simultaneous detection of two cell populations by two-colour fluorescence and application to the recognition of B-cell determinants. Nature 1976:262:795-7.

15 Mauff G. Alper CA. Awdeh Z, et al. Statement on the nomenclature of human C4 allotypes. Immunobiology 1983; 164: 184-91

16 Woolf $\mathrm{B}$. On estimating the variation between blood groups and disease. Ann Hum Genet 1955:19:251-3.

17 Haldane JBS. The estimation and significance of the logarithm of a ratio of frequencies. Ann Hum Genet 1955; 20:309-11.

1* Awdeh ZL, Raum D, Yunis EJ. Alper CA. Extended HLA/ complement allele haplotypes: evidence for T/t-like complex in man. Proc Natl Acad Sci USA 1983;80:259-63.

14 Baur MP. Danilovs JA. Population analysis of HLA-A, B, C, DR and other genetic markers. In: Terasaki PI. ed. Histocompatibility testing. Los Angeles: UCLA Tissue Typing Laboratory, 1980:955-93.

21) Bengtsson BO. Thomson G. Measuring the strength of associations between HLA antigens and disease. Tissue Antigens 1981:18:356-63.

21 Woodrow JC. Genetic aspects of the spondyloarthropathies. In: Panayi GS, ed. Seronegative spondyloarthropathies. Clinics in Rheumatic Diseases. Vol 11, No 1. London: Saunders, 1985:1-24.

22 Arnett FC. Bias WB. HLA-Bw38 and Bw39 in psoriatic arthritis: relationships and implications for peripheral and axial involvement. Arthritis Rheum 1980;23:649-50A.

${ }^{23}$ Murray C, Mann DL, Gerber LN, et al. Histocompatibility alloantigens in psoriasis and psoriatic arthritis. $J$ Clin Invest 1980:60:670-5.

${ }^{24}$ Beaulieu AD. Roy R. Mathon G, et al. Psoriatic arthritis: risk factors for patients with psoriasis-a study based on histocompatibility antigen frequencies. J Rheumatol 1983; 10:633-6.

${ }^{25}$ McKendry RJR. Sengar DPS, DesGroseilliers JP, Dunne JV. Frequency of HLA antigens in patients with psoriasis or psoriatic arthritis. Can Med Assoc J 1984:130:411-5.

26 Armstrong RD, Panayi GS. Welsh KI. Histocompatibility antigens in psoriasis, psoriatic arthropathy and ankylosing spondylitis. Ann Rheum Dis 1983:42:142-8.

Correspondence and requests for reprints to Professor J C Woodrow, University Department of Medicine, Royal Liverpool Hospital, PO Box 147, Liverpool L69 3BX. 